\title{
Theoretical-Probability Model for Calculating Roofness in Magnetic Abrasive Machining
}

\author{
Alexey Ikonnikov ${ }^{1, *}$ and Sergey Leonov ${ }^{1}$ \\ ${ }^{1}$ I.I. Polzunov Altai State Technical University, 656038 Barnaul, 46 Lenin Avenue, Russian \\ Federation
}

\begin{abstract}
The work is devoted to the problem of calculating the surface roughness during magnetic abrasive processing. Cutting grains have random dimensional characteristics, are randomly located on the surface of the tool, the workpiece has an irregular profile. The cutting parts of the grains partially remove the chips and partially elastoplastically deform the metal. Some of the vertices fall into the marks on the surface of the workpiece formed by the previous processing, and some - on the marks from the passage of the previous vertices. This process is determined by the probability of contact of the top of the grain with the metal. The developed probabilistic-theoretical model makes it possible to predict the removal of metal from the treated surface depending on the time and parameters of the operation, which creates the prerequisites for their use in the design of polishing operations.
\end{abstract}

\section{Introduction}

During magnetic-abrasive processing, grains, compacted by a magnetic field, interact with the treated surface, forming its profile. Due to the stochastic nature of the interaction, the resulting profile is also random. The characteristics of this profile and the roughness parameters obtained as a result of processing are determined by the distribution of grain sizes, cutting tops on them, the location of these tops over the surface of the workpiece, the original profile of the workpiece, and the force factor of interaction. In addition, a number of deterministic factors, such as the properties of the processed material, the characteristics of the tool (inductor), the parameters of the cutting mode, etc., affect the formation of the profile of the machined surface.

To predict the roughness obtained during magnetic abrasive machining, it is necessary to take into account the interaction of these stochastic parameters and deterministic parameters and their influence on the microcutting process, which forms the profile of the machined surface [1-4].

Modeling the machining process usually involves creating a mathematical description of three subsystems, the first of which characterizes the technological system, the second characterizes the cutting process, and the third one characterizes the output function, which

\footnotetext{
*Corresponding author: iamagtu@mail.ru
} 
forms the output indicators of the process. With regard to magnetic abrasive machining, the input parameters of the technological system are:

- initial roughness of the workpiece;

- physical and mechanical properties of the processed material;

- parameters of cutting conditions;

- properties of the tool (geometric parameters of the tool and the parameters of the magnetic abrasive powder) [5-7].

The structural model of the magnetic abrasive machining process is shown (Fig. 1).

The phenomena in the contact zone of the tool (inductor) and the workpiece are determined by the cutting process subsystem. It is this subsystem that forms the result of processing - the profile of the processed surface, which is formed due to the removal of metal from the surface of the workpiece.

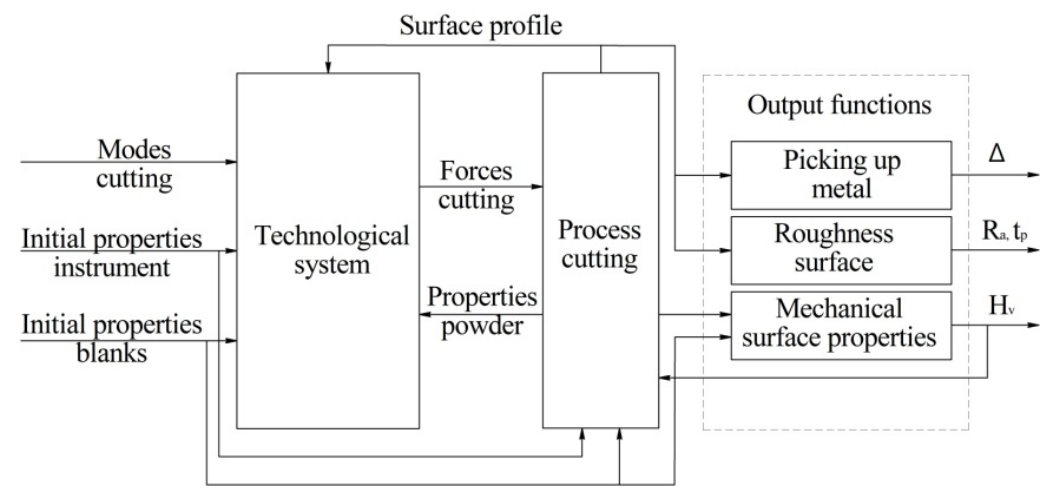

Fig. 1. Structural model of the magnetic abrasive machining process.

Magnetic abrasive grains combine magnetic and abrasive properties and have a different number of corners with randomly distributed radii. The depth of grains penetration into the workpiece material depends on these radii, as well as on the cutting force and microhardness of the workpiece [8].

The cutting parts of the tops of the grains partially remove the chips, and partially elastically-plastically deform the metal. The resulting risks are superimposed on the already created profile. Therefore, when the grain passes the contact zone of the tool with the workpiece, the cutting part of the grain contacts the metal only part of the time. Some of the vertices fall into the marks on the surface of the workpiece, formed by the previous processing, and some - into the marks from the passage of the previous vertices $[9,10]$. This process is determined by the probability of contact of the top of the grain with the metal.

\section{Research methodology}

Stochastic modeling of the process of forming the profile of the processed surface is based on the analytical transformation of the distribution functions of the random parameters of the interaction of the cutting tops of the grains with the processed surface into the distribution function of the ordinates of the profile of the processed surface. The approach used somewhat increases (in comparison with the Monte Carlo method) the complexity of the dependences obtained, sometimes requiring the use of numerical methods, approximation, and simplification of the dependences [11, 12, 13].

The analytical approach is in fact the opposite of the Monte Carlo method. Its main advantage is high performance: the distribution law and its parameters are obtained on the 
basis of analytical dependencies without the use of numerous cyclic calculations. This can take a significant amount of time to analyze dependencies. However, the high speed of the expressions obtained as a result of using the analytical approach makes its use preferable.

\section{Results and discussion}

Magnetic abrasive machining is commonly used to polish surfaces after finishing contour milling.

Ball milling cutters are most often used for milling complex-profile surfaces. The roughness profile depends on the cutter radius and cross feed $\mathrm{S}$ (Fig. 2).

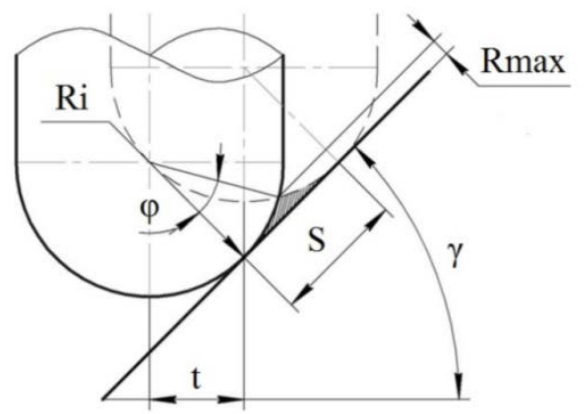

Fig. 2. Formation of microroughnesses during milling.

Taking into account the plastic deformation of the metal during milling, the shape of the resulting notch is set by the power dependence

$$
\mathrm{u}=\mathrm{ax} \text {. }
$$

In this case, the maximum risk height

$$
\mathrm{R}_{\max }=\mathrm{a}(\mathrm{S} / 2)^{\mathrm{b}} \text {. }
$$

To describe the surface profile during abrasive processing, Yu.K. Novoselov [14, 15] proposed to use the probability of material removal. Depending on the level y and the processing time $\tau$, this probability is calculated by the formula:

$$
\mathrm{P}_{\mathrm{M}}(\mathrm{y}, \tau)=1-\exp \left(-\mathrm{a}_{0}(\mathrm{y}, \tau)-\mathrm{a}_{1}(\mathrm{y})\right)
$$

where $a_{0}=k_{c} \lambda b_{3}$ characterizes the formation of a profile in the contact zone of the tool with the workpiece;

$a_{1}$ - characterizes the surface profile of the workpiece before processing;

$\mathrm{k}_{\mathrm{c}}$ - chip formation coefficient;

$\lambda$ - is the number of tops of grains that have passed a given section of the workpiece;

$b_{3}$ - is the average value of the grain width at the level of the conditional outer surface of the workpiece;

$\tau$ - time.

For a workpiece, $\mathrm{a}_{0}=0$ and the probability of material removal, which describes its profile, is determined by the dependence $P_{M}(y)=1-\exp \left(-a_{1}(y)\right)$.

With magnetic abrasive machining, metal removal occurs mainly from the top of the marks and the roughness introduced by the process is initially significantly less than the height of the marks after milling. In fact, we can assume that the top of the risks is cut off 
and the resulting profile has the form shown in (Fig. 3). In this case, the roughness of the horizontal platform is initially significantly less than the roughness of the entire surface.

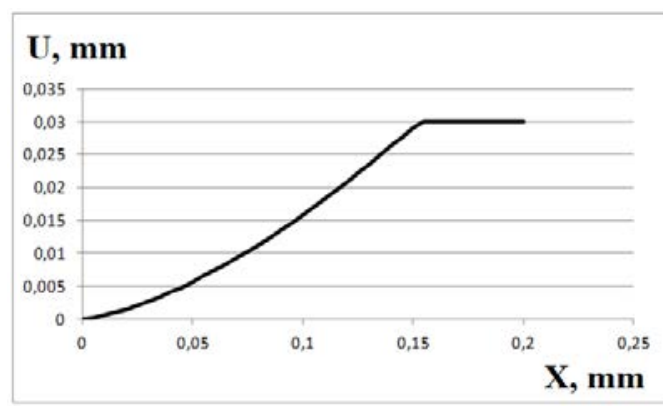

Fig. 3. Form the risks after removing a part of the metal.

The height of the risks is reduced by the amount of metal removal $\Delta$, which is determined taking into account the probability of material removal $\mathrm{P}_{\mathrm{M}}(\mathrm{y}, \tau)$. The roughness of the resulting surface is calculated by integrating the risk profile:

$$
R_{a}=\frac{2}{S} \int_{0}^{S / 2}|u(x)-W| d x,
$$

where $\mathrm{W}$ is the coordinate of the profile midline.

Analytically calculating the integral in expression (4) for the risks of (Fig. 3), we obtain the formula for calculating the roughness parameter $\mathrm{Ra}$ :

$$
\begin{gathered}
R_{a}=\frac{2}{s}\left(W\left(2 x^{*}-x_{1}\right)-\frac{a}{b+1}\left(2\left(x^{*}\right)^{b+1}-x_{1}^{b+1}\right)+\left(u_{\max }-W\right)\left(S / 2-x_{1}\right)\right), \\
W=\frac{2}{S} \int_{0}^{S / 2} u(x) d x=\frac{2}{S}\left[\frac{a}{b+1} x_{1}^{b+1}+\left(a\left(\frac{S}{2}\right)^{b}-\Delta\right)\left(\frac{s}{2}-x_{1}\right)\right] .
\end{gathered}
$$

where $x_{1}=\left((S / 2)^{b}-\Delta / a\right)^{1 / b}$;

$\Delta$ - metal removal;

$\mathrm{a}$ and $\mathrm{b}$ - risk parameters (1).

Fig. 4 shows the change in Ra with increasing $\Delta$.

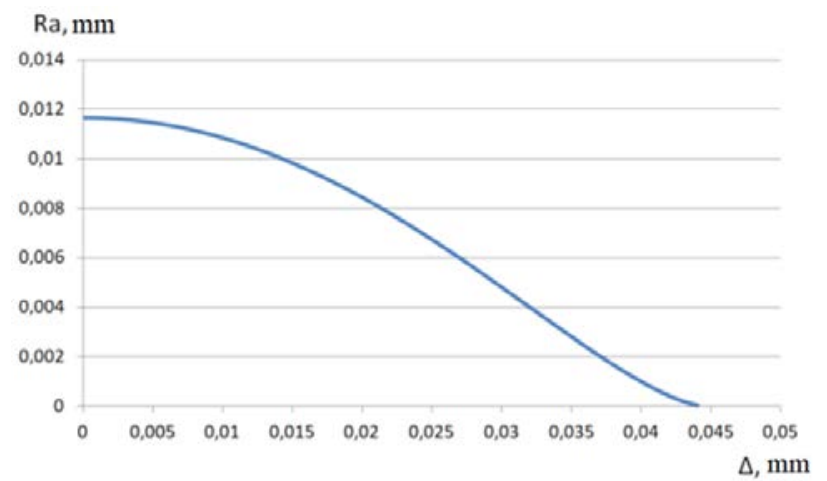

Fig. 4. Change in roughness when removing from the top of the risk.

In fact, the roughness value will tend not to zero, as in the graph in (Fig. 4), but some positive value, determined by the process of magnetic abrasive processing. 
To determine the introduced roughness, let us consider the process of interaction between the tool and the workpiece at discrete moments of time $\tau_{\mathrm{K}}$, corresponding to the exit of the magnetic circuit with grains from the contact with the workpiece. In this case, the value of the probability of contact (3) is determined by the coefficient $a_{0}\left(y, \tau_{\mathrm{K}}\right)$. Assuming that the grain tops are distributed in accordance with the density $f(u)=\frac{1,5}{t_{F}^{1,5}} \sqrt{u}$, and the profile of the grain top is described by the dependence $b_{z}=2 \sqrt{2 \rho\left(t_{F}-y-u\right)}$, the expression for of this coefficient at the moment of the exit of the magnetic circuit from contact with the workpiece $\tau_{\mathrm{K}}$ (Fig. 5) takes the form:

$$
\mathrm{a}_{0}\left(\mathrm{y}, \tau_{\mathrm{K}}\right)=\frac{3 \pi k_{c} n_{z}}{8 t_{F}^{1.5}}\left(t_{F}-y\right)^{2} L_{p} \sqrt{2 \rho}
$$

where $t_{F}$ is the maximum cutting depth, $n_{z}$ is the number of grains per unit surface of the tool, $\rho$ is the average radius at the top of the grain, $\mathrm{k}_{\mathrm{c}}$ is the chip formation coefficient.

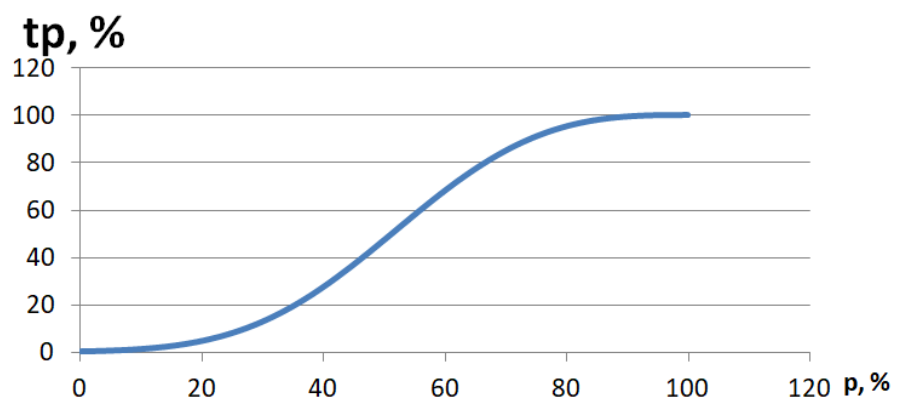

Fig. 5. Relative reference length of the profile.

The relative reference profile length $t_{\mathrm{p}}$ is determined by the values of the metal removal probability. In fact, $t_{p}=(1-\mathrm{P}(\mathrm{y})) 100 \%$. In this case, the argument $t_{p}$ is also the relative level, expressed as a percentage: $\mathrm{p}=\left(\mathrm{y} / \mathrm{t}_{\mathrm{F}}\right) 100 \%$.

Multipass is considered to calculate the probability of material removal. In this case, the probability of metal removal in a steady-state processing mode is calculated by the formula:

$$
P(y)=1-\exp \left(-Q \sum_{k=0}^{m}\left(t_{\phi}-y-k \Delta\right)^{2}\right) .
$$

To calculate the height parameter of roughness $\mathrm{Ra}$, you can use the formula:

$$
R a=\int_{0}^{W}(1-P(y)) d y+\int_{W}^{t_{\phi}} P(y) d y
$$

where $\mathrm{W}$ is the coordinate of the midline: the level $\mathrm{y}$ at which $\mathrm{P}(\mathrm{y})=\mathrm{t}_{\mathrm{p}}=0.5$.

Expressing these dependences in dimensionless form, we obtain a system of equations that must be solved numerically:

$$
\left\{\begin{array}{c}
m=\left[\frac{1-p}{X}\right] ; \\
p=1-\frac{b+\sqrt{b^{2}-4 a c}}{2 a} .
\end{array}\right.
$$

where $\mathrm{X}=\Delta / \mathrm{t}_{\mathrm{F}}$;

$\mathrm{a}=\mathrm{m}+1$;

$\mathrm{b}=\mathrm{Xm}(\mathrm{m}+1)$;

$\mathrm{c}=X^{2} \frac{m(m+1)(2 m+1)}{6}-F_{1}$.

$\mathrm{W}=\mathrm{pt}_{\mathrm{F}}$. 
After that, the roughness of the treated surface is calculated by the formula

$$
R a=\frac{1}{\pi Q \sum_{k=0}^{m}(W-k \Delta)},
$$

Calculation example:

Let $\mathrm{F}_{1}=19.21581 ; \mathrm{X}=0.026008 ; \mathrm{t}_{\mathrm{F}}=0.0003 \mathrm{~mm} ; \mathrm{Q}=12674311 / \mathrm{mm}^{2}$.

Solving the system of equations (10) by the simple iteration method, we obtain: $\mathrm{p}=$ $0.512141 ; \mathrm{m}=249 ; \mathrm{W}=1.54 \mathrm{e}-05 \mathrm{~mm}$. In this case, $\mathrm{Ra}=1.03 \mathrm{E}-05 \mathrm{~mm}=0.0103$ microns.

For approximate calculations, instead of solving the system of equations (10), we can assume $\mathrm{p}=0.5$.

\section{Conclusions}

1. The analytical approach to constructing stochastic models can significantly reduce the time for predicting the quality indicators of magnetic abrasive machining, providing the necessary accuracy of calculations.

2. Magnetic abrasive machining is most often used for polishing milled surfaces across the lines. In this case, metal removal mainly occurs from the tops of the scratches until their height is comparable to the roughness introduced by the polishing itself.

3. The developed stochastic models make it possible to predict the roughness of the processed surface depending on the time and parameters of the operation, which creates the prerequisites for their use in the design of polishing operations.

\section{References}

1. Biing-Hwa Yana, Geeng-Wei Changa, Tsung-Jen Chenga, Rong-Tzong Hsub, Journal of Machine Tools \& Manufacture, 43, 1355 (2003)

2. Dhirendra K. Singh, V.K. Jain, V. Raghurama, R. Komanduri, Wear 259, 1254 (2005) 1254-1261.

3. Dhirendra K. Singh, V.K. Jain, V. Raghuram, Journal of Materials Processing Technology, 149. 22 (2004)

4. B. Girma B, S.S. Joshi, M.V. Raghuram, R. Balasubramaniam, Machine Sci Technology, 10 (3), 32334 (2006).

5. Harish Kumar, Sehijpal Singh, Pardeep Kumar, Journal Of Engineering Research \& Technology (IJERT), 2, 3, (2013)

6. S.C. Jayswal, V. K. Jain, P.M. Dixit, International Journal of Advanced Manufacturing Technology, 26, 477 (2005)

7. Junmo Kanga, Andrew Georgea, Hitomi Yamaguchia, Procedia CIRP 1414 (2012)

8. K. Singh, Dhirendra \& Jain, Vijay \& Raghuram, V.. Journal of Materials Processing Technology, 149, 22, (2004)

9. Krishankant, Jatin Taneja, Mohit Bector, Rajesh Kumar, International Journal of Engineering and Advanced Technology (IJEAT), 2, 1, (2012)

10. Baljinder Singh, International Research Journal of Engineering and Technology (IRJET), 2, 04 (2015)

11. Hitomi Yamaguchia, Anil K. Srivastavab, Michael A. Tana, Journal of Manufacturing Technology, 61, 311 (2012) 
12. T.C.Kanish, P.Kuppan, S.Narayanan, S. Denis Ashok, Procedia Engineering, 97, 1948 (2014).

13. E.Y. Tatarkin, A.M. Ikonnikov, T.A. Schreiner, R.V. Grebenkov, Applied Mechanics and Materials, 788, 69 (2015)

14. Y. Novoselov, V. Bogutsky., L. Shron, Procedia Engineering Cep. "International Conference on Industrial Engineering, ICIE 2017", 991 (2017)

15. Y. Novoselov, V. Bogutsky, L. Shron, A. Kharchenko, MATEC Web Of Conferences Cep. "International Conference on Modern Trends in Manufacturing Technologies and Equipment, ICMTMTE 2017", 01080 (2017) 